Laser smoothing of sub-micron grooves in hydroxyl-rich fused silica

N. Shen, M. J. Matthews, J. E. Fair, J. A. Britten, H. T. Nguyen, D. Cooke, S. Elhadj, S. T. Yang

November 16, 2009

Applied Surface Science 
This document was prepared as an account of work sponsored by an agency of the United States government. Neither the United States government nor Lawrence Livermore National Security, LLC, nor any of their employees makes any warranty, expressed or implied, or assumes any legal liability or responsibility for the accuracy, completeness, or usefulness of any information, apparatus, product, or process disclosed, or represents that its use would not infringe privately owned rights. Reference herein to any specific commercial product, process, or service by trade name, trademark, manufacturer, or otherwise does not necessarily constitute or imply its endorsement, recommendation, or favoring by the United States government or Lawrence Livermore National Security, LLC. The views and opinions of authors expressed herein do not necessarily state or reflect those of the United States government or Lawrence Livermore National Security, LLC, and shall not be used for advertising or product endorsement purposes. 


\title{
Laser smoothing of sub-micron grooves in hydroxyl-rich fused silica
}

\author{
Nan Shen, Manyalibo J. Matthews, James E. Fair, Jerry A. Britten, Hoang T. Nguyen, Diane \\ Cooke, Selim Elhadj, and Steven T. Yang \\ Lawrence Livermore National Laboratory, \\ 7000 East Avenue, L-491, Livermore, CA 94550-9234 USA
}

\begin{abstract}
Nano- to micrometer-sized surface defects on UV-grade fused silica surfaces are known to be effectively smoothed through the use of high-temperature localized $\mathrm{CO}_{2}$ laser heating, thereby enhancing optical properties. However, the details of the mass transport and the effect of hydroxyl content on the laser smoothing of defective silica at submicron length scales is still not completely understood. In this study, we examine the morphological evolution of sub-micron, dry-etched periodic surface structures on type II and type III $\mathrm{SiO}_{2}$ substrates under $10.6 \mu \mathrm{m} \mathrm{CO}_{2}$ laser irradiation using atomic force microscopy (AFM). In-situ thermal imaging was used to map the transient temperature field across the heated region, allowing assessment of the $\mathrm{T}$ dependent mass transport mechanisms under different laser-heating conditions. Computational fluid dynamics simulations correlated well with experimental results, and showed that for large effective capillary numbers $\left(\mathrm{N}_{\mathrm{c}}>2\right)$, surface diffusion is negligible and smoothing is dictated by capillary action, despite the relatively small spatial scales studied here. Extracted viscosity values over 1700 -2000K were higher than the predicted bulk values, but were consistent with the surface depletion of $\mathrm{OH}$ groups, which was confirmed using confocal Raman microscopy.
\end{abstract}




\section{Introduction}

Thermal modification of nano- and micro-structures on a wide variety of material surfaces to create or enhance surface properties using long-wave $\mathrm{CO}_{2}$ lasers has enjoyed significant success in recent decades $[1,2]$. Recently, localized $\mathrm{CO}_{2}$ laser heating of silica glass has been successfully applied to control and mitigate surface damage on optics used in high power laser applications such as inertial confinement fusion (ICF) [3-5]. In particular, minimizing damage initiation and shot-to-shot damage growth on UV-grade optics surfaces which have been mechanically polished [6], laser machined [7] or optically damaged [8] is crucial to enhancing overall laser performance. However, the physics of the apparent extrinsic damage threshold of fused silica surfaces is still a subject of debate, and therefore the mechanism by which this threshold is enhanced by $\mathrm{CO}_{2}$ laser heating is also not completely clear. Because the nano- and micro-scale fractured surfaces associated with polishing/machining are believed to be associated with a higher concentration of UV-absorbing damage precursors [9, 10], thermal treatment may act to allow these defects to anneal by way of surface or bulk diffusion. On the other hand, enhancement of local field intensity from irregular interfaces, clearly present on optically damaged and laser machined surfaces, could also act to lower the damage threshold of laser optics $[11,12]$. In this case, $\mathrm{CO}_{2}$ laser heating would act to macroscopically smooth these surfaces into more regular interfaces, thus lowering near-field intensification effects. Therefore, the exact role and contribution from each of these mechanisms and the optimization of $\mathrm{CO}_{2}$ laser surface treatment from a fundamental point of view, remains somewhat unclear. It is of interest therefore to explore the temperature dependence of the reshaping of model surfaces to 
understand the relative contributions from capillarity and diffusion, as well as influence from structural-chemical changes, to the mitigation of specific defects associated with UV absorption.

Previous theoretical studies of the isothermal relaxation of modulated surfaces under capillary, evaporative and diffusive processes can be traced back to the pioneering work by Mullins nearly four decades ago [13, 14]. For an arbitrary surface decomposed into Fourier components $\omega=2 \pi / \lambda$, where $\lambda$ is the spatial period, the relative importance of each transport process to the evolution of a nearly-flat surface of height $S(x, y, t)$ can be described by

$$
\frac{\partial S}{\partial t}=-\left(F \omega+A \omega^{2}+D \omega^{3}+B \omega^{4}\right) S
$$

where $F, A, D$, and $B$ are the decay constants for capillary flow, evaporation-condensation, bulk diffusion, and surface diffusion respectively. Although for the relaxation of nanoscale surface features both surface diffusion and viscous flow should be the dominant transport processes, Wang et al. [15] recently concluded that, for isothermal heating of sub-micron Al-Ca-Si oxide gratings, the effect of surface diffusion is negligible. One possible explanation the author attributed to the observation is the existence of defects which reduces surface diffusion [16], though the mechanism of surface diffusion on highly defective ceramic surfaces is still poorly understood. A similar, yet somewhat empirical, approach derived for borosilicate thin films [17] was used by Nowak et al. [18] to study laser polishing of micron-scale features in fused silica. A decay function, similar to that in Eq.(1) was used to fit data, but deviation in the expected spatial power scaling was left unresolved. Because laser materials processing of roughened and defective surfaces is subject to a dynamic and non-uniform temperature field, it is not clear what 
mechanisms are at play, particularly at the relatively shorter time scales and higher transient temperatures characteristic of laser processing.

It is well-known that $\mathrm{OH}$ content plays a significant role in determining the thermomechanical properties of fused silica glass, particularly viscosity. For example, in the low water content regime $(<0.1 \mathrm{wt} \%)$, water exits mainly as structurally bound and immobile hydroxyl groups [19], yielding viscosities (in Poise) of $\log \eta \sim 14$ at 1400K. In contrast, high water content silica can contain more loosely bound and mobile $\mathrm{OH}$ groups and $\mathrm{H}_{2} \mathrm{O}$, leading to viscosities of $\log \eta \sim 12$ at 1400K. One model developed by Adam and Gibbs [20] to understand the mechanism by which water affects glass melts quantifies the rate of structural relaxation as a function of both kinetic and thermodynamic parameters. The fundamental idea of the model is that the relaxation of a liquid to an equilibrium configuration following a change in external conditions is limited not only by local bond strengths (i.e., Si-O), but also by the number of configurations the liquid can access at any particular temperature, which is enhanced by $\mathrm{OH}$ mobility. Therefore, the introduction of different amounts of $\mathrm{OH}$ content in silica glass leads to variation in configurationally entropy and the heat capacity of the glass, affecting their viscosity.

In this work, we simulate the process of viscous glass flow using computational fluid dynamics (CFD) software and compare it to the measured decay kinetics of semi-square submicron gratings etched on fused silica glass with different $\mathrm{OH}$ content. Direct measurement of the transient temperature field and changes in near-surface glass structure and composition are used to estimate viscosities and relaxation times associated with grating decay. As surmised in previous studies, depletion of $\mathrm{OH}$ from the surface, confirmed by Raman microscopy, leads to a shift in viscosity to higher values than predicted for the bulk. However, for large effective 
capillary numbers, $N_{c}$, i.e. shorter time scales $(<1 \mathrm{~s})$ and lower temperatures $(<1700 \mathrm{~K})$, this shift is less dramatic, and may indicate an enhancement of surface relaxation caused by either surface diffusion or an incomplete near-surface depletion of $\mathrm{OH}$ species.

\section{Sample preparation and experimental procedure}

We used two types of fused silica glass in the study, Corning 7980 (type III, 800 1000ppm OH content by weight) and Heraeus Suprasil 312 (type II 200ppm OH content by weight), which we will refer to as samples $\mathrm{C}-3$ and $\mathrm{H}-2$ respectively. These samples were chosen primarily because of their relatively wide use in UV-grade laser optics, and their disparate $\mathrm{OH}$ levels. One-dimensional trapezoidal gratings with a period of $\sim 650 \mathrm{~nm}$ and a height of $\sim 700 \mathrm{~nm}$ were patterned on 51mm round, $10 \mathrm{~mm}$ thick, silica samples using e-beam lithography and ion-beam etching [21] as shown in Fig. 1. Grating shapes were nearly isosceles, with grating tops $\sim 250 \mathrm{~nm}$ wide with RMS roughness of $\sim 1 \%$. Patterned silica samples were exposed to focused CW $10.6 \mu \mathrm{m} \mathrm{CO}_{2}$ laser light from a Synrad firestar V20, with a maximum output power of 20 watts and power stability of $\pm 5 \%$. The laser beam profile was nearly a perfect

Gaussian a $1 / e^{2}$ diameter of $\sim 1 \mathrm{~mm}$, as characterized by a beam profiler (Pyrocam III, Spiricon). The laser power delivered to the sample was set to between 3.8 and $4.7 \mathrm{~W}$, while the exposure time was adjusted from 100ms to 100s. This narrow power range was chosen to observe surface relaxation over reasonable experimental time spans, while also staying well below the evaporation threshold of $~ 3000 K[4]$. During each laser exposure, the sample surface temperature was monitored in-situ by using a calibrated LN-cooled HgCdTe camera operating at 33fps, with an optical resolution of $\sim 100 \mu \mathrm{m}$ (sampled at $40 \mu \mathrm{m}$ spacing). Although this spatial resolution is 
quite coarse relative to the grating geometry, it can be shown that, in fact, the calculated temperature profile established by a $1 \mathrm{~mm}$ beam varies quite slowly over the length scales studied here. Further details of the laser exposure setup and thermographic analysis can be found in Ref. [22]. The surface morphology of each laser exposed region of the etched samples was characterized before and after exposure using a Digital Instrument Dimension 3100 atomic force microscope (AFM). We used high aspect ratio silicon tips (Veeco OTESPAW) to ensure the accuracy of our AFM results, and to minimize any tip convolution of the shapes measured. Instrument resolution was $<1 \mathrm{~nm}$ vertically and $\sim 10 \mathrm{~nm}$ laterally. Finally, spatially-resolved variations in $\mathrm{OH}$ content caused by laser heating were measured using confocal Raman microscopy operating at 532nm (CW), with a spatial (depth) and spectral resolution of $5 \mu \mathrm{m}$ and

$4 \mathrm{~cm}^{-1}$ respectively.[23]. Our detection limit of $\mathrm{OH}$ content was estimated to be roughly 100ppm by weight.

\section{Simulation of the surface relaxation}

The surface evolution of the 2D periodic step during laser heating was simulated using a commercial computational fluid dynamics code optimized for free surface flow [24]. Because our goal was to use the simulation as a basis for comparison with experiment, explicit viscous stress and surface capillary pressure evaluations were used to ensure maximum accuracy of the numerical solution. Taking as a characteristic velocity $U=W / t$, where $\mathrm{W}$ is the grating period, $\mathrm{t}$ the exposure time, and evaluating Reynolds Number, Weber Number and Froude Number ratios to determine the relative roles of inertial, viscous, capillary and gravitational forces we find all 
these ratios are «1: $\mathrm{N}_{\mathrm{Re}}=2 \times 10^{-15}(1 / \mathrm{t}), \mathrm{N}_{\mathrm{We}}=9 \times 10^{-15}\left(1 / \mathrm{t}^{2}\right), \quad \mathrm{N}_{\mathrm{Re}} /\left(\mathrm{N}_{\mathrm{Fr}}\right)^{2}=2.2 \times 10^{-8}(\mathrm{t})$, and $\mathrm{N}_{\mathrm{We}} /$ $\left(\mathrm{N}_{\mathrm{Fr}}\right)^{2}=8.6 \times 10^{-8}$

Hence, with typical flow times of 1-100 seconds and in the non-evaporative regime, only viscous and capillary forces are important in determining surface reshaping and material transport. Unfortunately, this problem resists solution by explicit algorithm because the small spatial scale in conjunction with large shear forces leads to a severe restriction in maximum computational time steps. For example, the maximum computation time step associated with viscous momentum transport for fused silica at $2000 \mathrm{~K}$ is limited to $\Delta t_{\min } \leqslant \rho \Delta x_{\min }^{2} / \eta \sim 10^{-16}$ sec, a very impractical value. However, by taking the ratio $\mathrm{N}_{\mathrm{Re}} / \mathrm{N}_{\mathrm{We}}$ we can define a non-dimensional relaxation variable $\tau$ (the inverse capillary number, $\mathrm{N}_{\mathrm{c}}$ ),

$$
\tau \equiv\left(\frac{W^{2} \rho}{\mu t}\right)\left(\frac{W^{3} \rho}{\sigma t^{2}}\right)^{-1}=\frac{\sigma t}{\mu W}=N_{c}^{-1}
$$

which gives the only required equivalence condition of formal dynamic similarity. We are therefore free in the numerical simulation to uniformly reduce surface tension and viscosity without influencing the essential character of the system. The only restriction is that we not decrease viscosity to the point where inertial effects become appreciable, because as previously established, inertia plays no significant role in this problem. This practical constraint is simply that $\mathrm{N}_{\mathrm{Re}}$ in the simulation remain below approximately 0.01 .

The flow of a periodic surface structure closely approximating those formed by photolithography (Fig. 1) for our study was simulated. Taking advantage of the inherent symmetry, only one half of the periodic structure was included in the calculation. A uniform 10nm finite difference grid was used with $90^{\circ}$ free surface contact angle constraints and free-slip, 
zero flux conditions imposed at domain boundaries. The simulation was performed using constant (local) viscosity and surface tension, consistent with $\mathrm{W}<<\ell_{\mathrm{th}}$ where $\ell_{\mathrm{th}} \sim 1 \mathrm{~mm}$ is the thermal diffusion length. The model geometry and results of the simulation are shown in Fig. 2, indicating snapshots of the surface profile as it evolves during the surface tension driven flow process. As shown, the surface assumes a sinusoidal profile at a dimensionless time between $\tau=1$ and $\tau=2$. It is evident that pure exponential decay of the step height commences around $\tau=2$. The early time behavior is consistent with the findings of Cassidy and Gjostein [25] who analyzed similar idealized flat top structures by the phenomenological theory of Mullins [13] based on Fourier decomposition of the surface profile into a set of independent decay modes. The long time behavior follows simple exponential decay corresponding to viscous relaxation of a pure sinusoidal surface under forces of capillary pressure [17]. Therefore, the time evolution of grating height $h(t)$ from an initial height $h_{0}$ can be reasonably approximated in these two regimes using two analytic functions of the form

$$
\frac{h(t)}{h_{0}}=C \exp \left(K \tau^{M}\right)
$$

where the parameters C, K, M for small $(\tau<2)$ and large $(\tau>2)$ values of $\tau$ are given in Table I. These analytic functions could then be used to directly fit experimental surface height data corresponding to surface treatments at varying temperatures and times.

\section{Results}


Due to the non-uniform heating inherent to laser materials processing, the spatial profile of the laser power translates into a variation in surface temperature resulting in an effective viscosity profile (Fig. 3). The resulting spatial non-uniformity in flow dynamics was probed by measuring the height change of the grating at different locations within one laser beam, and correlating each spatial subset of data with in situ surface temperature measurements. Figure 3a shows a microscope image of sample C-3 treated with a 3.8W, 100s exposure, with square boxes indicating radial sub-regions that were scanned using AFM. For comparison, the Gaussian laser beam profile and the resulted spatial distribution of grating heights were illustrated with schematics in Fig. 3a. High-resolution AFM scans along the radius of the exposed regions separated by $25 \mu \mathrm{m}$ were taken for each case studied, as shown in the upper graph of Fig. 3b. Simultaneous to the laser exposure, 2D thermographic images were taken using the HgCdTe camera, which could be spatially registered with subsequent AFM scans, as indicated by the temperature profile shown in the lower panel of Fig. 3b. The peak on-axis temperature ranged from 1140 to $1640 \mathrm{~K}$ and 1340 to $1960 \mathrm{~K}$ for the $3.8 \mathrm{~W}$ and $4.7 \mathrm{~W}$ exposures respectively, with the temperature rise scaling with exposure time $100 \mathrm{~ms}<\mathrm{t}<100$ s roughly as $\sqrt{\mathrm{t}}$. Although the steadystate temperature profile is known to vary as a Bessel-weighted Gaussian [26], near the peak we use a simple Gaussian fit to extract the temperature variation, as indicated in Fig. 3b.

As described in Sec. III, the surface flow dynamics are expected to depend principally on the viscosity, which, for a wide range of glasses and temperatures follows the so-called VogelFulcher-Tammann (VFT) expression [27],

$$
\eta=\eta_{0} \exp \left(\frac{B_{0}}{T-T_{0}}\right)
$$


where $\eta_{0}, B_{0}$ and $T_{0}$ are temperature-independent constants. However, since we are studying the behavior of glass flow in a relatively narrow temperature range (1700 2000K), the VFT equation can be well-approximated by an Arrhenius relation, $\eta=\eta_{0} \exp \left(E_{a} / R T\right)$, where $E_{a}$ is the activation energy of viscous glass flow and $\mathrm{R}$ is the gas constant. The values of $\eta_{0}$ and $E_{a}$ have been reviewed and summarized for a wide range of $\mathrm{OH}$ containing $\mathrm{SiO}_{2}$ samples by Zandian [28]. The C-3 sample is a type III glass containing $1000 \mathrm{ppm}$ OH corresponding to a reported value of $\eta_{0}=8.81 \times 10^{-5}$ Poise and $E_{a}=434 \mathrm{~kJ} /$ mole. Applying these values into our expression for viscosity for the 3.8W, 100s exposure condition and comparing it with our CFD simulation, a significant discrepancy was found between simulation and experiment (Fig. 4). In the experiment, since the laser exposure time and local surface temperature are known for each sub-region studied within the treated regions, measurement of the grating morphology changes can, in fact, be used to estimate local viscosity [25]. By using the Arrhenius approximation for viscosity in the expression for $\tau$ (Eq.2), and fitting $h(t) / h_{0}$ as a function of T for a single case (3.8W/100s), we could obtain $\eta_{0}$ and $E_{a}$ for sample C-3 under $\mathrm{CO}_{2}$ laser polishing conditions (Fig. 5a). Using the same approach, we also calculated $\eta_{0}$ and $E_{a}$ for sample H-2 (Fig. 5b). Table II summarizes the values of the activation energy for the two types of silica glass found in the literature and measured under our experimental conditions. The extracted viscosity was then applied to all laser exposure conditions for sample C-3 and H-2. A reasonable agreement between the experiment and simulation is achieved with several different laser parameters when we used this new Arrhenius expression for viscosity (Fig. 6). However, for small $\tau$ (short exposure times and lower temperatures), there existed a deviation in the data away from the curve indicating a faster relative decay rate. 


\section{Discussion}

For capillary-driven flow, viscosity limits the relaxation processes near glass transition temperature. Therefore knowing the viscosity of a glass is important to predicting processing results. Direct measurement of the viscosity of glass at high temperature is often difficult, especially under extreme, non-uniform conditions such as $\mathrm{CO}_{2}$ laser mitigation. Study of the relaxation of grating structures may provide an attractive method to indirectly fine-tune the temperature dependant viscosity at high temperature. It is worth noting that the non-dimensional parameter $\tau$ gives some insight on the relationship between time and viscosity (temperature) during laser polishing. When designing methods for polishing a feature of size $w$, it is possible to achieve smoothing by optimizing either the transient exposure time $t$ or temperature to reach a desired viscosity.

Although we simplified the problem of surface relaxation by considering only the heat activated surface tension driven mass flow, the model adequately describes the $\mathrm{CO}_{2}$ laser smoothing of sub-micron sized $\mathrm{SiO}_{2}$ gratings for most of the temperature/time scales examined here. The computational fluid dynamics simulation based on this model afforded us the ability to treat exactly realistic (e.g. trapezoidal) gratings of any aspect ratio, without the constraints typical of the nearly-flat plane solution $[13,25]$. In particular, deviation from a pure exponential decay can be observed due to relaxation of higher spatial frequency components. For example, the spatial power scaling $\mathrm{M}$ shown in Table II was found to be $1-1.35$ for our geometry, similar to the values found by Nowak et al. for larger scale surface features [18]. It is important to point out that, although a power scaling $\mathrm{M}>1$ might imply the contribution of other mechanisms 
through Eq.(1), capillary flow of high aspect ratio, non-sinusoidal geometries leads to a similar effect.

A key consideration here with regard to capillarity is the proper temperature dependent viscosity, which for silica is strongly dependent on $\mathrm{OH}$ content [28]. The viscosity extracted from our simulation and experiments for both high and low $\mathrm{OH}$ samples for $\tau$ larger than $\sim 0.5$ $\left(\mathrm{N}_{\mathrm{c}}<2\right)$ indicated a shift in $\boldsymbol{E}_{\boldsymbol{a}}$ towards higher values. The higher activation energy $E_{a}$ could suggest that the hydroxyl content may have diffused out during laser heating. The apparent increase in viscosity due to an assumed $\mathrm{OH}$ diffusion was reported by Wang et al., and though highly plausible, only bulk $\mathrm{OH}$ was actually measured [15]. Indeed, given the smaller starting $\mathrm{OH}$ content, it is interesting to note that the viscosity shift for the lower $\mathrm{OH}$ sample $(\mathrm{H}-2)$ was somewhat less. In order to explore laser heating-induced chemical changes that may correspond to higher activation energies estimated from our surface smoothing results, we performed spatially-resolved confocal Raman microscopy to monitor the $\mathrm{OH}$ variation on the sample surface and in bulk. The normalized intensity of the SiOH stretch at $\sim 3700 \mathrm{~cm}^{-1}$ has been shown to give a reasonable estimate of the amount of $\mathrm{OH}$ [23], given the spectra can be calibrated with a known OH sample. Bulk infrared transmission measurements of sample H-2 were performed by the vendor [29] and used to calibrate $\mathrm{OH}$ changes detected by Raman for both samples. Figure 7 shows the $\mathrm{OH}$ variation as a function of depth (axial resolution $\sim 5 \mu \mathrm{m}$ ) for each sample type, for different exposure times. The estimated bulk OH content 900ppm for sample C-3 was consistent with quoted vendor specs (800 1000ppm). As shown, a clear OH depletion region is evident for sample C-3, consistent with the relative increase in viscosity predicted by our relaxation analysis. As expected, when exposure time is decreased from 100 to 1 s, less $\mathrm{OH}$ is depleted from the C-3 sample, a 200ppm decrease as opposed to 400ppm. On the other hand, 
the $\mathrm{H}-2$ sample showed much less of an effect, though the low starting $\mathrm{OH}$ content and relative sensitivity of our measurement ( 100ppm) may limit our ability to assess $\mathrm{OH}$ depletion for this sample. It is interesting to note that the variation in the $\mathrm{OH}$ content of sample C-3 at the surface for different laser parameters may explain some of the scatter in $h / h_{0}$ vs $\tau$ below $\tau=0.5$ shown in Fig. 4: while the viscosity was estimated using a heavily depleted case ( $\mathrm{t}=100 \mathrm{~s})$, shorter times may retain more bulk-like $\mathrm{OH}$ levels and hence viscosity closer to that of the bulk. On the other hand, a stronger decay of $h / h_{0}$ could also point to a possible contribution from surface diffusion, not accounted for in our model. Although this deviation was not as strong in sample $\mathrm{H}-2$, thus supporting a possible non-uniform $\mathrm{OH}$ effect, further studies are planned to elucidate this apparent discrepancy for small $\tau$ in high $\mathrm{OH}$ samples.

\section{Conclusions}

The smoothing of nanometer-scale, dry-etched periodic surface structures on type II and type III $\mathrm{SiO}_{2}$ substrates with $10.6 \mu \mathrm{m} \mathrm{CO}_{2}$ laser has been studied using atomic force microscopy (AFM). In-situ thermal imaging was used to map the transient temperature field across the heated region, allowing assessment of the T-dependent mass transport mechanisms and surface reshaping under different laser-heating conditions. Computational fluid dynamics simulations correlated well with experimental results, and showed that capillary-driven flow dominates, even at the relatively small spatial scales studied here. The transient local viscosity of the silica glass appears to deviate from typical literature values, consistent with a depletion of $\mathrm{OH}$ content that varies with exposure conditions. However, at shorter time scales $(<1 \mathrm{~s})$ and lower temperatures 
$(<1700 \mathrm{~K})$, this shift is less dramatic, and may indicate an enhancement of surface relaxation caused by either surface diffusion or an incomplete near-surface depletion of $\mathrm{OH}$ species. Direct measurement of the $\mathrm{OH}$ content using micro-Raman confirmed these general conclusions, though the possible influence of surface diffusion in altering relaxation behavior remains somewhat unclear and is a subject of future study.

\section{Acknowledgements}

The authors would like to thank Drs. Bill Henshaw and Michael Feit for useful discussions on UV light scattering, and Dr. James Stolken for help in assessing silica viscosity data. This work performed under the auspices of the U.S. Department of Energy by Lawrence Livermore National Laboratory under Contract DE-AC52-07NA27344 


\section{References}

[1] Duley, W. W., "Co2 Lasers, Effects and Applications." New York: Academic, 1976.

[2] Dahotre, N. B., Lasers in surface engineering. Materials Park, OH: ASM International, (1998).

[3] Bass, I. L., Draggoo, V. G., Guss, G. M., Hackel, R. P., and Norton, M. A., "Mitigation of laser damage growth in fused silica NIF optics with a galvanometer scanned CO2 laser - art. no. 62612A," presented at Conference on High-Power Laser Ablation VI, Taos, NM, 2006.

[4] Mendez, E., Nowak, K. M., Baker, H. J., Villarreal, F. J., and Hall, D. R., "Localized CO2 laser damage repair of fused silica optics," Applied Optics, vol. 45, pp. 5358-5367, (2006).

[5] Palmier, S., Gallais, L., Commandre, M., Cormont, P., Courchinoux, R., Lamaignere, L., Rullier, J. L., and Legros, P., "Optimization of a laser mitigation process in damaged fused silica," Applied Surface Science, vol. 255, pp. 5532-5536, (2009).

[6] Campbell, J. H., "Damage resistant optical glasses for high power lasers: A continuing glass science and technology challenge," Glass Science and Technology, vol. 75, pp. 91108, (2002).

[7] Prasad, R. R., Bruere, J. R., Peterson, J., Halpin, J., Borden, M., and Hackel, R. P., "Enhanced performance of large of optics using UV and IR lasers," Laser-Induced Damage in Optical Materials: 2003, vol. 5273, pp. 288-295 540, (2003).

[8] Demos, S. G., Staggs, M., and Kozlowski, M. R., "Investigation of processes leading to damage growth in optical materials for large-aperture lasers," Applied Optics, vol. 41, pp. 3628-3633, (2002).

[9] Laurence, T. A., Bude, J. D., Shen, N., Feldman, T., Miller, P. E., Steele, W. A., and Suratwala, T., "Metallic-like photoluminescence and absorption in fused silica surface flaws," Applied Physics Letters, vol. 94, pp. -, (2009).

[10] Gallais, L., Natoli, J. Y., and Amra, C., "Statistical study of single and multiple pulse laser-induced damage in glasses," Optics Express, vol. 10, pp. 1465-1474, (2002).

[11] Genin, F. Y., Salleo, A., Pistor, T. V., and Chase, L. L., "Role of light intensification by cracks in optical breakdown on surfaces," Journal of the Optical Society of America aOptics Image Science and Vision, vol. 18, pp. 2607-2616, (2001).

[12] Ge, D. B. and Zhu, X. Q., "Simulation of a class of optical scattering with interaction between obstacle and interface," presented at 8th World Multi-Conference on Systemics, Cybernetics and Informatics, Orlando, FL, 2004.

[13] Mullins, W. W., "Flattening of a nearly place solid surface due to capillarity," Journal of Applied Physics, vol. 30, pp. 77-83, (1959).

[14] Mullins, W. W., "Theory of Thermal Grooving," Journal of Applied Physics, vol. 28, pp. 333-339, (1957). 
[15] Wang, L. L., Ellison, A. J. G., and Ast, D. G., "Investigation of surface mass transport in Al-Si-Ca-oxide glasses via the thermal induced decay of submicron surface gratings," Journal of Applied Physics, vol. 101, pp. -, (2007).

[16] Naumovets, A. G., "Collective surface diffusion: An experimentalist's view," Physica aStatistical Mechanics and Its Applications, vol. 357, pp. 189-215, (2005).

[17] White, L. K., Miszkowski, N. A., Kurylo, W. A., and Shaw, J. M., "Flow Properties and Contour Modeling of Fusible Borophosphosilicate Glasses," Journal of the Electrochemical Society, vol. 139, pp. 822-826, (1992).

[18] Nowak, K. M., Baker, H. J., and Hall, D. R., "Efficient laser polishing of silica microoptic components," Applied Optics, vol. 45, pp. 162-171, (2006).

[19] Davis, M. J., "The effect of water on the viscosity of silicate melts: A configurational entropy approach," vol. 63, pp. 167-173, (1999).

[20] Adam, G. and Gibbs, J. H., "On Temperature Dependence of Cooperative Relaxation Properties in Glass-Forming Liquids," vol. 43, pp. 139-\&, (1965).

[21] Britten, J. A., Molander, W., Komashko, A. M., and Barty, C. P. J., "Multilayer dielectric gratings for Petawatt-class laser systems," presented at 35th Annual Symposium on Optical Materials for High-Power Lasers, Boulder, CO, 2003.

[22] Yang, S. T., Matthews, M. J., and Elhadj, S., "Thermal transport in CO2 laser irradiated fused silica: in situ measurements and analysis " in press, Journal of Applied Physics, (2009).

[23] Mikkelsen, J. C., Galeener, F. L., and Mosby, W. J., "Raman Characterization of Hydroxyl in Fused-Silica and Thermally Grown Sio2," Journal of Electronic Materials, vol. 10, pp. 631-651, (1981).

[24] Flow Science, I., "Flow3D ${ }^{\mathrm{TM}}$ V9.2 ". Harkle Rd Ste A, Santa Fe, NM 87505.

[25] Cassidy, D. C. and Gjostein, N. A., "Capillarity-Induced Smoothing of Glass Surfaces by Viscous Flow," Journal of the American Ceramic Society, vol. 53, pp. 161-\&, (1970).

[26] Lax, M., "Temperature Rise Induced by a Laser-Beam," Journal of Applied Physics, vol. 48, pp. 3919-3924, (1977).

[27] Doremus, R. H., "Viscosity of silica," Journal of Applied Physics, vol. 92, pp. 76197629, (2002).

[28] Zandian, V., Florry, J. S., and Taylor, D., "Viscosity of Fused-Silica with Different Hydroxyl Contents," British Ceramic Transactions and Journal, vol. 90, pp. 59-60, (1991).

[29] Heraeus, private communication. 


\section{TABLES}

Table I. Function parameters used to approximate viscous relaxation behavior.

\begin{tabular}{|c|c|c|c|}
\hline & $\mathrm{C}$ & $\mathrm{K}$ & $\mathrm{M}$ \\
\hline$\tau<2$ & 1.00 & 0.80 & 1.35 \\
\hline$\tau>2$ & 3.14 & 1.57 & 1.00 \\
\hline
\end{tabular}

Table II. Viscous flow activation energies for different fused silica glass samples.

\begin{tabular}{|c|c|c|c|c|}
\hline \multirow{2}{*}{ Sample } & \multicolumn{2}{|c|}{ Arrhenius fitting parameters } & \multicolumn{2}{c|}{ Reported value } \\
\cline { 2 - 5 } & Pre-exponential & $\begin{array}{c}\text { Activation energy } \\
(\mathrm{kJ} / \text { mole) }\end{array}$ & Pre-exponential & $\begin{array}{c}\text { Activation energy } \\
(\mathrm{kJ} / \mathrm{mole})\end{array}$ \\
\hline Corning 7980 & $3 \times 10^{-9}$ & 603 & $8.81 \times 10^{-5}$ & 434 \\
\hline Suprasil 312 & $2.05 \times 10^{-8}$ & 578 & $5.8 \times 10^{-7}$ & 515 \\
\hline
\end{tabular}




\section{FIGURE CAPTIONS}

Figure 1: Typical AFM images of grating (a) before and (b) after $\mathrm{CO}_{2}$ laser polishing.

Figure 2: Simulated grating height evolution with analytical fit.

Figure 3: Spatial map of the region where surface profiles of the grating were measured using AFM and the corresponding temperature at that location. (a) Microscopy image of region treated with the Gaussian $\mathrm{CO}_{2}$ laser beam; (b) the grating height at different locations relative to laser beam center and the in-situ temperature measurement.

Figure 4: Experiential (open circle) and simulation (straight line) results of normalized grating height vs. $\tau$. The values of $\tau$ was calculated using Arrhenius relation $\eta=\eta_{0} \exp \left(E_{a} / R T\right)$ for

literature reported viscosity for silica glasses with $\sim 1100 \mathrm{OH}$ content where $\eta_{0}=8.81 \times 10^{-5}$ Poise and $E_{a}=434 \mathrm{~kJ} / \mathrm{mole}$.

Figure 5: Arrhenius temperature dependent viscosity relation $\eta=\eta_{0} \exp \left(E_{a} / R T\right)$ obtained by fitting experiment data for (a) type III (C-3) and (b) type II (H-2) silica glasses.

Figure 6: Experimental (open symbols) and simulation (solid line) of the evolution of the grating height vs. $\tau$. For (a) type III (C-3), the laser parameters are 3.8W 10s ( $\square)$, 3.8W 100s (O), 4W 100s (*), 4.2W 10s $(\nabla), 4.5 \mathrm{~W}$ 5s $(\triangle)$, 4.5W 30s (\#), 4.7W 1s $(\oplus)$ and 4.7W 10s $(\diamond)$. For (b) type II (H-2) silica glass, the laser parameters are 3.8W 10s $(\square)$, 4.5W 10s $(\triangle)$.

Figure 7: Measured $\mathrm{OH}$ concentration as a function of depth for 1s (closed symbols) and 100s (open symbols) exposures, for samples C-3 (triangles) and $\mathrm{H}-2$ (circles). 
(a)
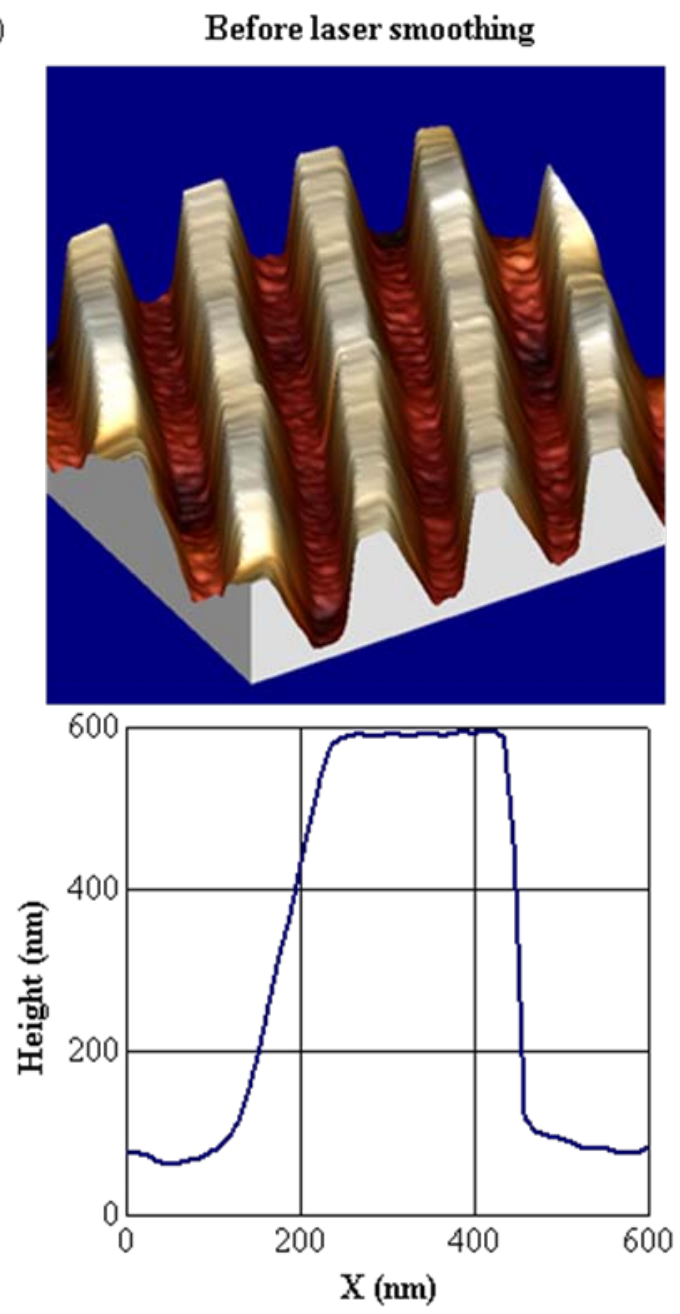

(b)
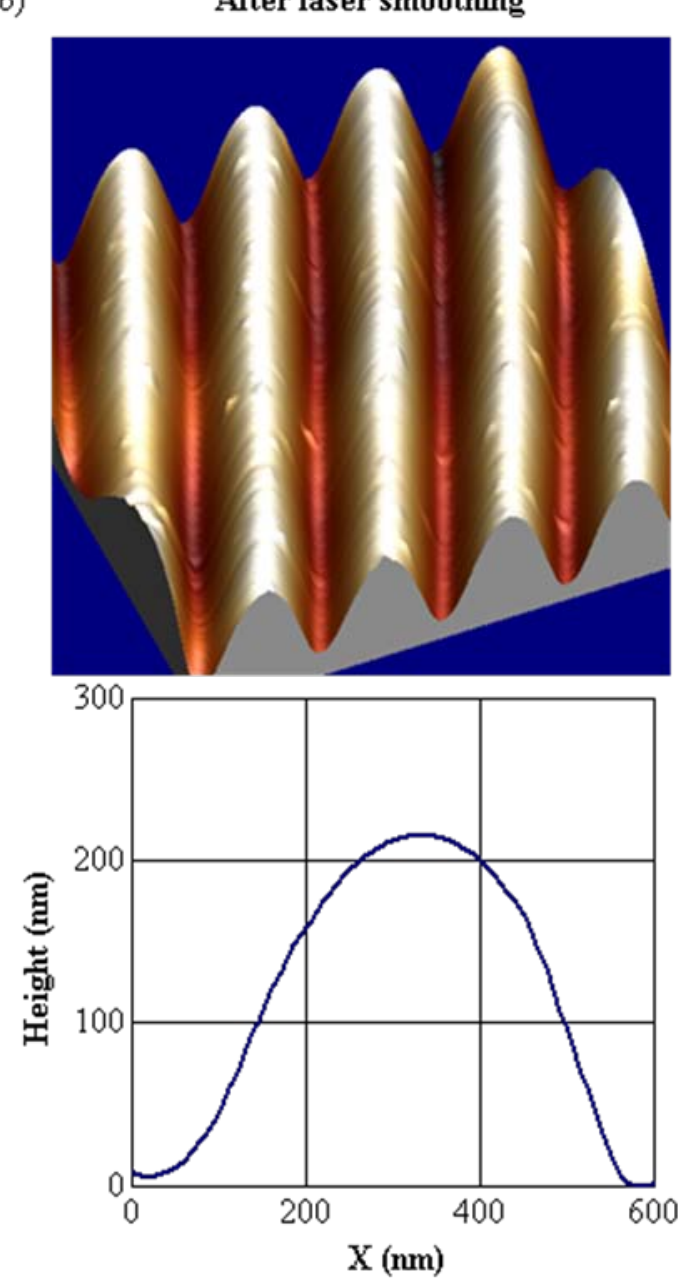

Figure 1 


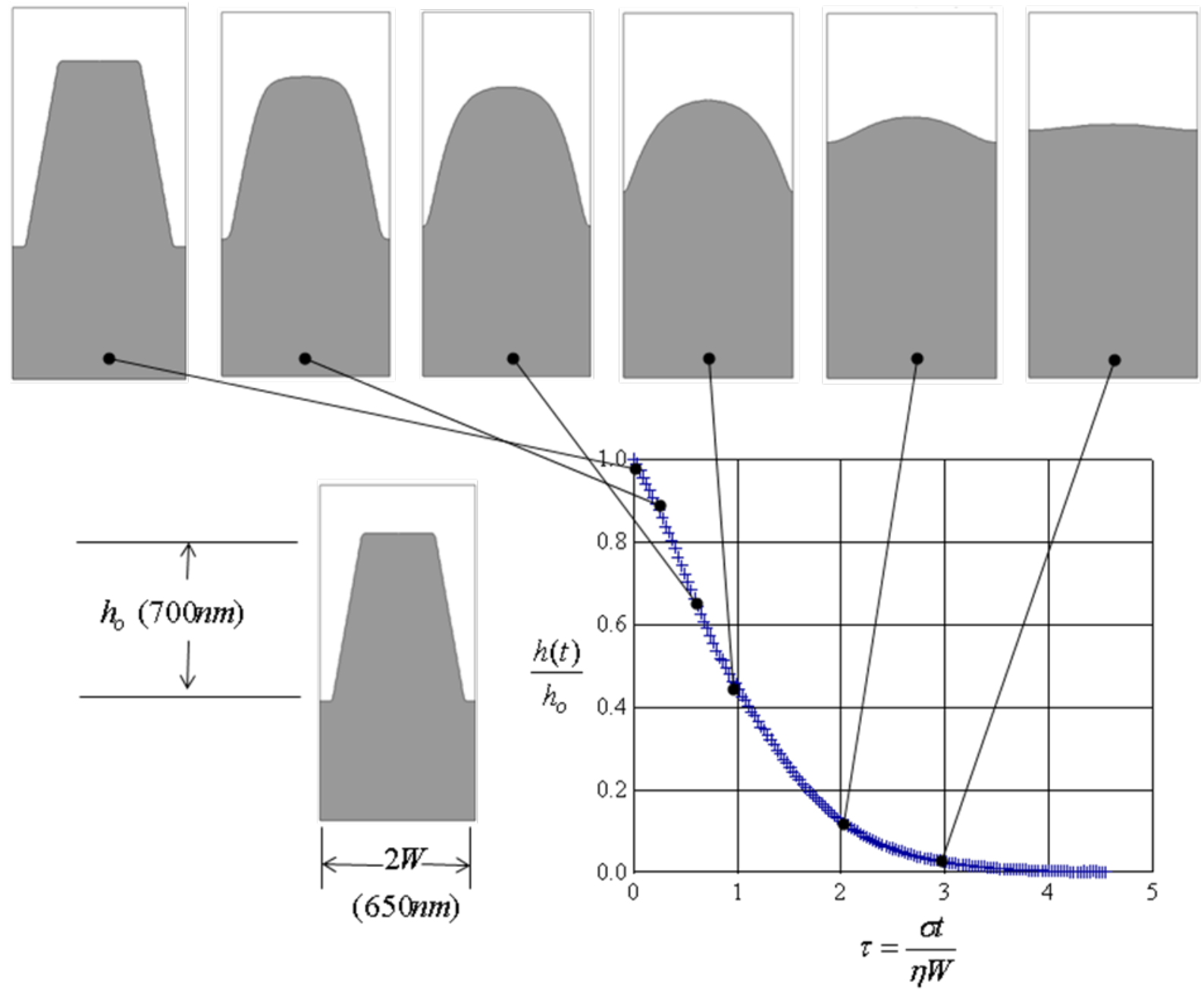

Figure 2 
(a)

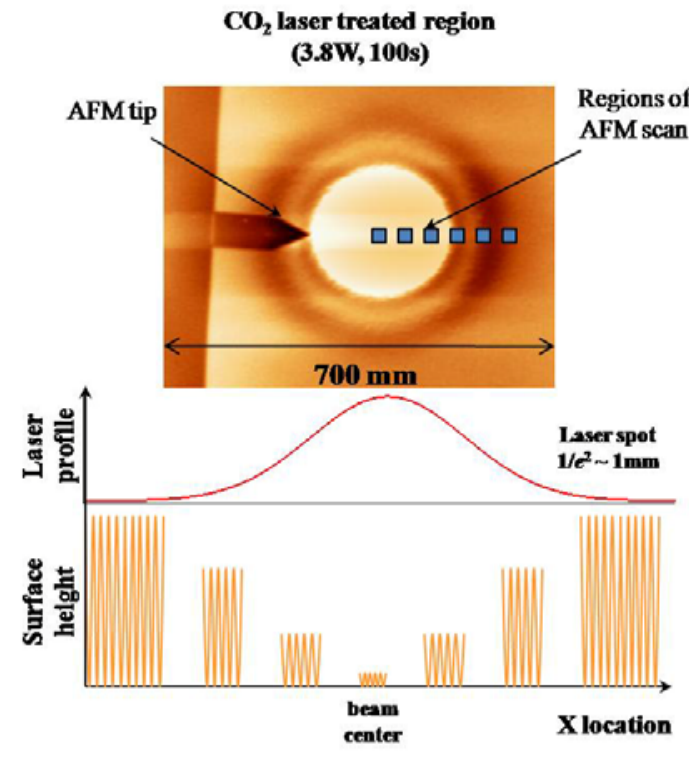

(b)

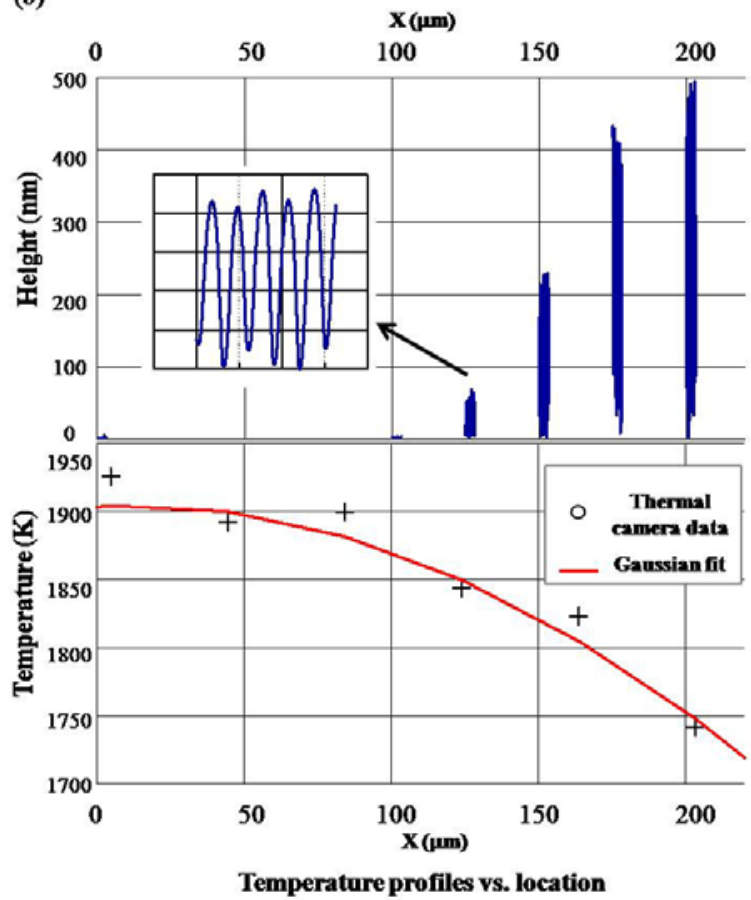

Figure 3 
Temperature (K)

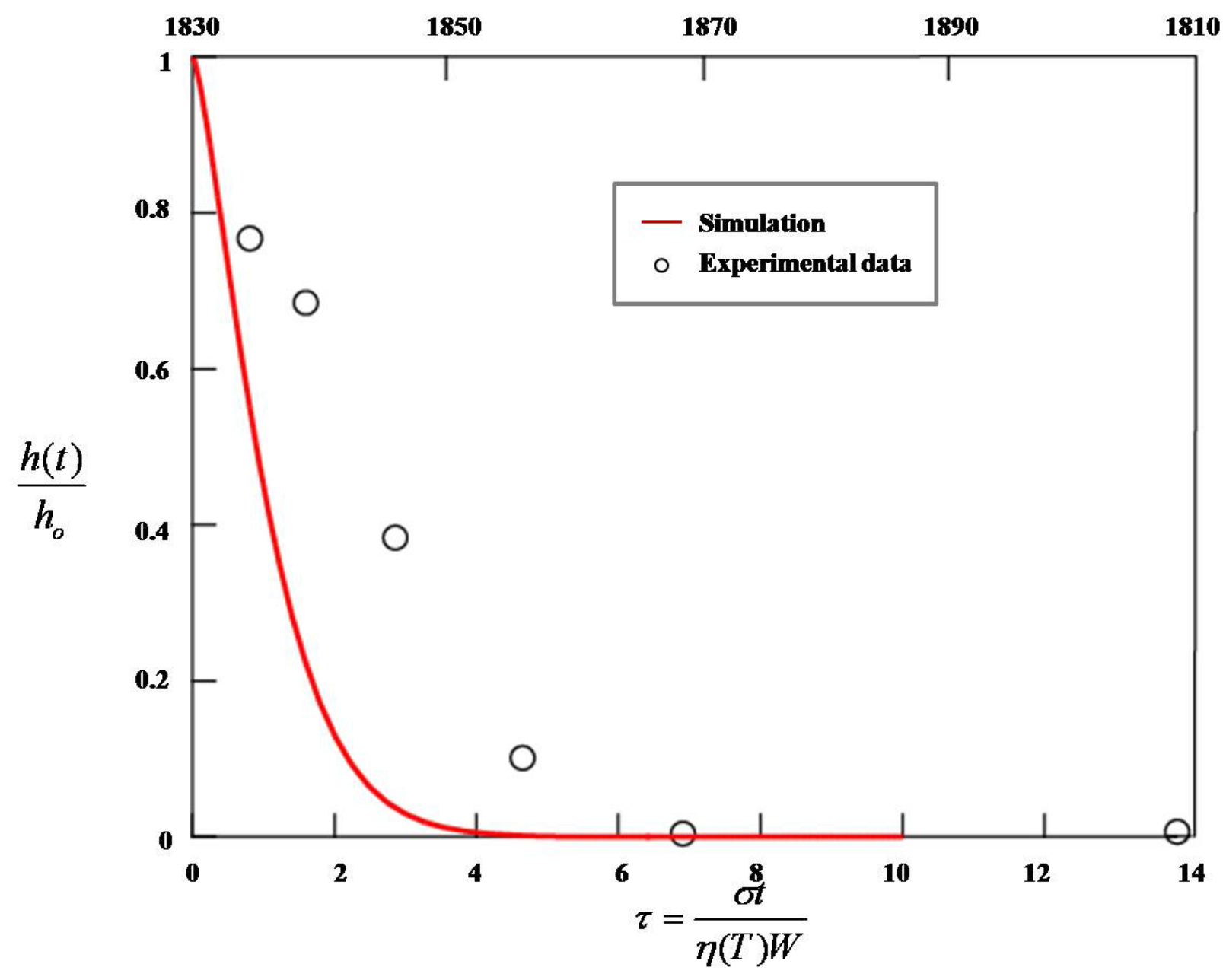

Figure 4 
(a)

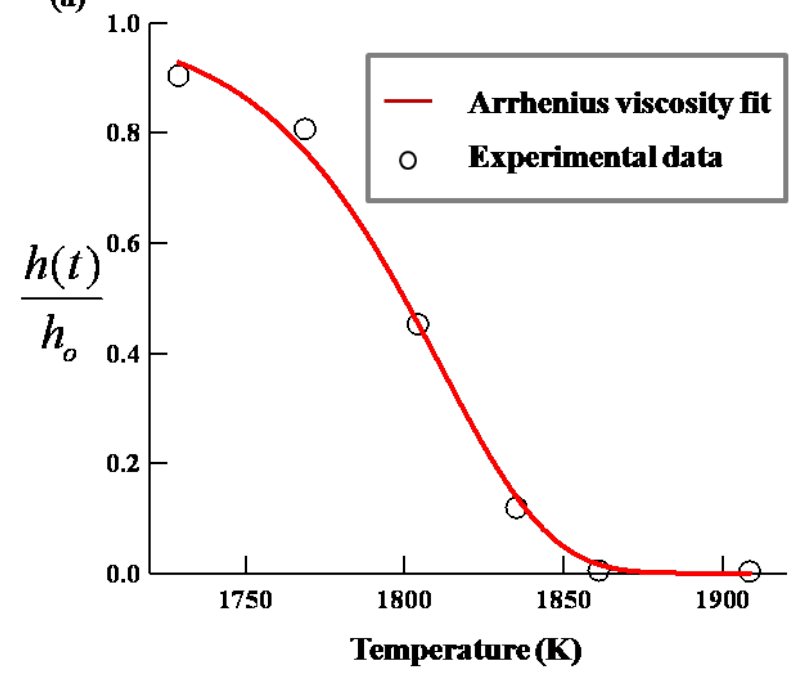

(b)

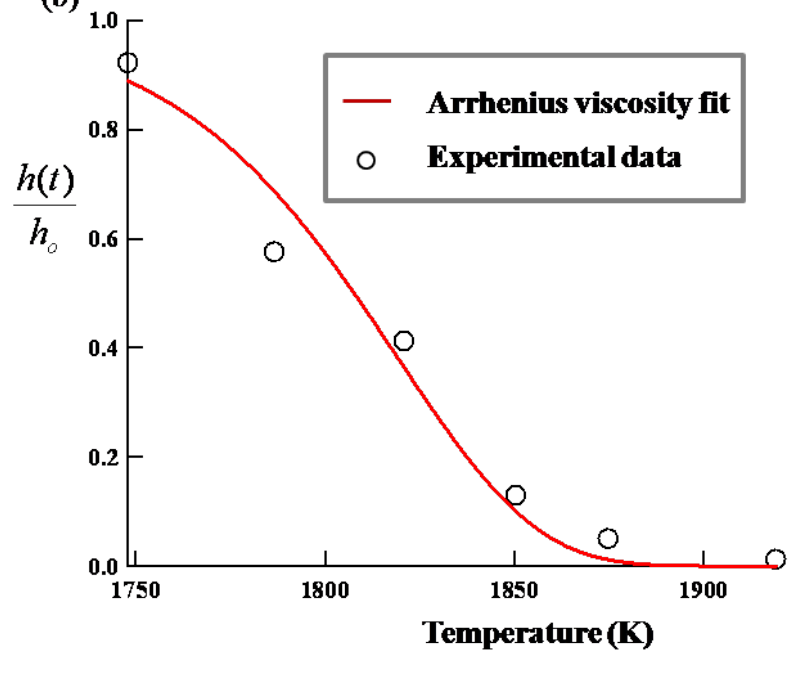

Figure 5 

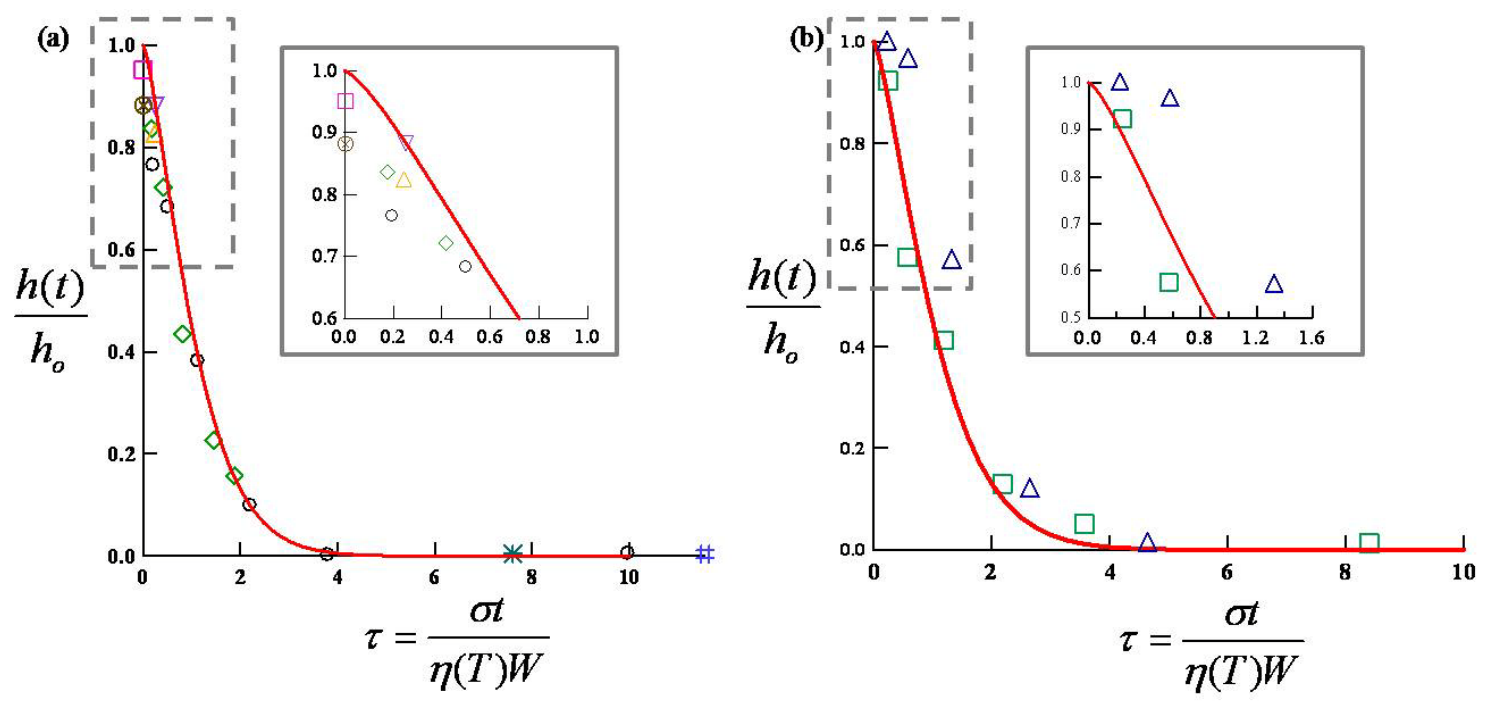

Figure 6 


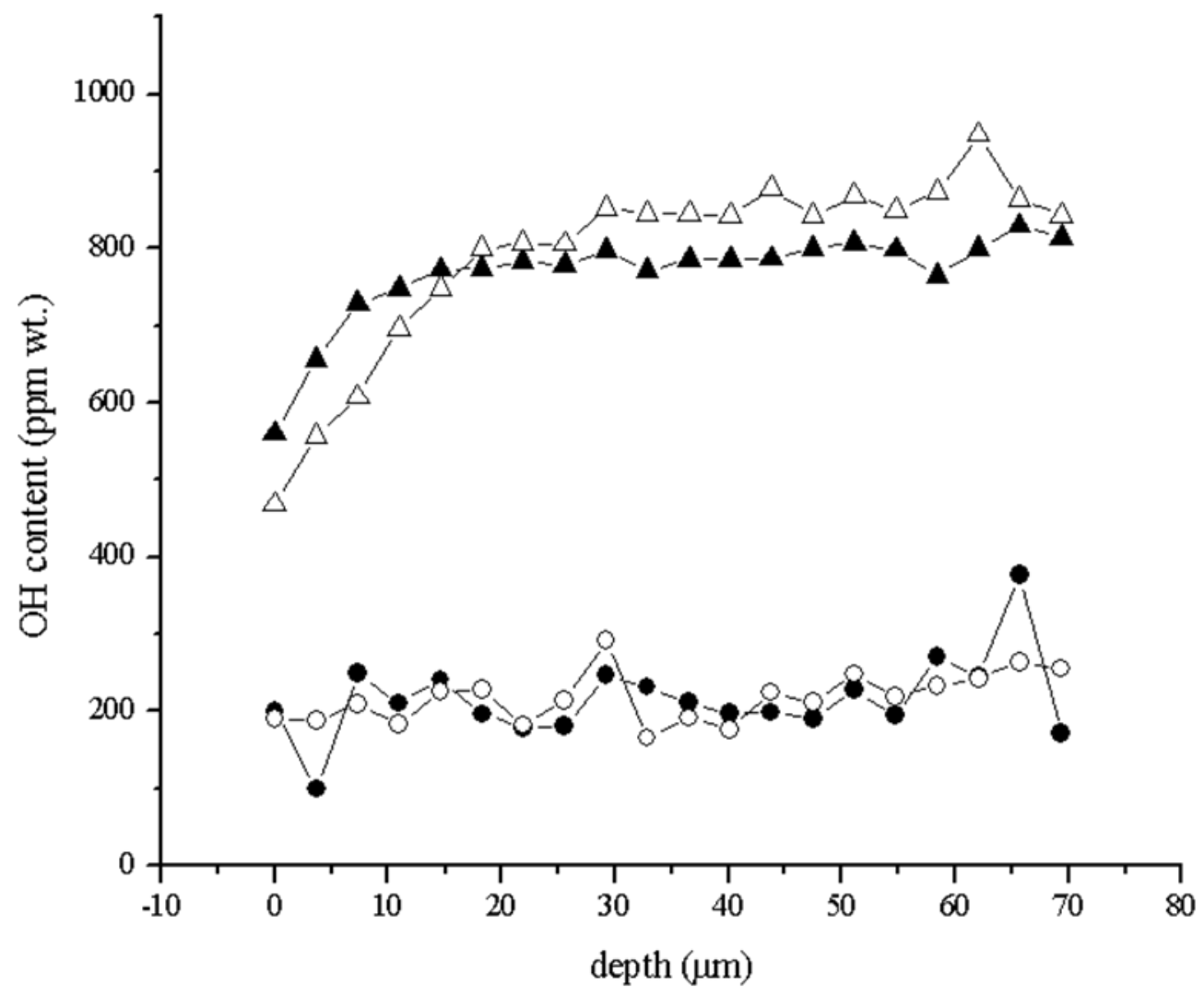

Figure 7 